\title{
An Examination of the Differences in Flow between Individual and Team
} Athletes
Authors' contribution:
A) conception and design of the study
B) acquisition of data
C) analysis and interpretation of data
D) manuscript preparation
E) obtaining funding

\author{
Joni M. Boyd ${ }^{1 \mathrm{~A}, \mathrm{~B}, \mathrm{D}}$, David P. Schary ${ }^{1 \mathrm{C}, \mathrm{D}}$, Andy R \\ Worthington ${ }^{1 \mathrm{~A}, \mathrm{~B}, \mathrm{D}}$, Seth E. Jenny ${ }^{2 \mathrm{~A}, \mathrm{C}, \mathrm{D}}$ \\ ${ }^{1}$ Winthrop University, USA \\ ${ }^{2}$ Slippery Rock University of Pensylwania, USA
}

ABSTRACT

The purpose of this exploratory study was to investigate differences between the flow experiences of NCAA Division I team athletes versus individual athletes. A volunteer sample of 104 collegiate athletes completed a 42-item flow questionnaire. Multiple linear regressions showed mean flow scores for team athletes were significantly higher than individual athletes $(\beta=-1.66, p=0.004)$, with an $\mathrm{R}^{2}$ value of 0.03 . The type of sport was a significant predictor of three of the nine dimensions of flow, with the largest difference explaining $9 \%$ of the variance. The results of this study are unique and answer the repeated call in the literature for team flow research. In summary, team sport athletes experienced total flow at a higher overall rate than individual sport athletes, allowing for the conclusion that team sport athletes can and KEYWORDS do experience flow.

flow, athlete, peak performance, in the zone

\section{Introduction}

The psychological factors that contribute to, or inhibit, a successful athletic performance continue to be an area of interest in the application of sport psychology. Research into these factors creates an understanding as to what psychological processes occur within sport performance. Investigations into the controllability of sport performance have used different variables, some more applicable than others. One factor of heightened interest is the state of flow in athletes, specifically its relationship to quality athletic performance. Csikszentmihalyi (1990) described flow as a state in which people are so immersed in an activity that nothing else matters. Often, flow is described as "being in the zone." Athletes desire to experience the state of flow because it is an "intrinsically rewarding" experience (Csikszentmihalyi 1990), and although it is found to occur most frequently in elite athletes, flow can occur across all levels of skill (Bakker, Oerlemans, Demerouti, Slot, \& Ali 2011). Jackson and Marsh (1996) further describe the flow state as a situation where personal skills equal or exceed the required challenges of the sport, and this experience is perceived as enjoyable, free from distractions, and typically leads to an overall good performance. Despite the interest in flow, there is still a need to understand how flow may differ between individual and team sport athletes because there is little research comparing flow experiences between individual and team athletes. 
One concept that is often misunderstood is the difference between flow and peak performance, as they are often used synonymously, but are different. Flow has been described as fun and enjoyable, and peak performance has been described as playful, fun, and fulfilling (Privette \& Bundrick 1991). Stavrou, Jackson, Zervas, and Karteroliotis (2007) state that the two cannot be used interchangeably, since someone could be in flow and may not actually experience peak performance. Flow in and of itself is an intrinsically rewarding experience, whereas peak performance is seen as one performing at an optimal level (Privette 1983; Privette \& Bundrick 1991). However, if one achieves peak performance, it is likely he or she was in the flow state (Csikszentmihalyi 1990). Jackson (1996) describes peak performance as a "standard of accomplishment rather than a psychological state" (p. 76). Thus, flow is often found to be a precursor for peak performance (Jackson 1988; Jackson \& Roberts 1992); however, peak performance is not a requirement for flow (Stavrou et al. 2007).

The majority of studies examining flow in sport have focused on individual sport because it has been shown to be more likely to elicit flow (Bakker et al. 2011). However, there is evidence that flow does occur in the team sport setting (Jackson 1995), but comparisons between the flow experiences of individual and team sport athletes have not been extensively examined (Chavez 2008; Russell 2001). Russell (2001) found empirical evidence of flow experienced across both team and individual settings, but the focus was on examining differences among gender or sport setting with regard to factors important to the flow state. To date, other than the Russell (2001) study, there is little known research comparing the experience of flow between individual and team sport athletes. In reaction to the few results from previous calls for research on team flow, Swann (2016) reemphasized the need for increased attention on team flow, stating that more research will help develop a more robust explanation of flow occurrence. Additionally, Swann (2016) noted the need for further research across sports, including those in individual and team competitions.

The calls for more research comparing the experience of flow between team and individual athletes is not without merit. It is possible that the dimensions of flow could vary between individual and team athletes. For example, the challenge-skills balance and autotelic experience dimensions of flow are believed to be experienced on a higher level in team athletes. This may happen because the challenge for a team is the opponent they face and the experience is often more enjoyable due to their interaction with each other. On the other hand, flow dimensions such as clear goals and unambiguous feedback are believed to be experienced on a higher level in individual athletes due to a greater reliance on themselves, as well as feedback from coaches to achieve their goals, because they do not have teammates to help them along in the process. Thus, the purpose of this study was to examine the differences in the experience of flow between athletes participating in individual sports and those participating in team sports.

\section{Method}

\section{Participants and Procedures}

The a prior power analysis was conducted using GPower to determine a sufficient sample size using an alpha of 0.05, a power of 0.80, and a medium effect size $\left(f^{2}=0.15\right.$; Faul, Erdfelder, Buchner, \& Lang 2013). Due to the lack of previous literature on the topic, the standard medium effect size for linear regression was used in the power analysis. Based on the aforementioned assumptions, the desired sample size was 85 . After participant recruitment, 104 Division I collegiate student-athletes from a university in southeastern United States participated in the study. Table 1 includes all the characteristics of the sample, which included 37 males (36\%) and 67 females (64\%). Ages ranged from 18 to 23 years old $(M=19.7, S D=1.3)$. Most participants were either freshman $(n=37)$ or juniors $(n=31)$. The majority of those completing the survey were Caucasian $(n=69)$ or African American $(n=16)$. Team sport athletes $(n=67)$ were represented by basketball, baseball, soccer, and volleyball. Individual sport athletes $(n=37)$ were represented by cross country, golf, tennis, and track and field. In addition, for this study it was assumed that the athletes had enough playing years to experience the flow state at least once. 
Table 1

Demographics $(n=104)$

\begin{tabular}{lc}
\hline Characteristics & $N(\%)$ or $M(S D)$ \\
\hline Gender & $37(35.5)$ \\
Male & $67(64.5)$ \\
Female & $19.7(1.3)$ \\
Age & \\
Year in school & $35(33.6)$ \\
Freshman & $19(18.3)$ \\
Sophomore & $31(29.8)$ \\
Junior & $19(18.3)$ \\
Senior or above & \\
Race/Ethnicity & $16(15.4)$ \\
African American & $69(66.3)$ \\
Caucasian & $19(18.3)$ \\
Other & \\
Sport & $37(35.5)$ \\
Individual & $67(35.5)$ \\
Team & \\
\hline Source: Own study & \\
\hline
\end{tabular}

Source: Own study.

Participants were recruited via emails to the athletic department, who forwarded the emails to all studentathletes. Willing participants completed a 45-item survey through a link in the emails. The survey was administered via Qualtrics software (Qualtrics, Provo, UT). Time to complete the survey was approximately five to ten minutes. Permission was obtained through the university's Institutional Review Board, and participants were given a written statement at the beginning of the surveys and directed to continue the survey if they consented to be in the study.

\section{Measures}

Flow was measured using the 36-item Flow State Scale (FSS; Jackson \& Marsh 1996). The FSS asks athletes to recall an experience of flow and answer questions based on that experience. The FSS contains nine dimensions that were formed in the original research of Csikszentmihalyi (1990). The flow dimensions (subscales) include the following:

1. Challenge-skills,

2. Action-awareness,

3. Clear goals,

4. Unambiguous feedback,

5. Concentration,

6. Sense of control,

7. Loss of self-consciousness,

8. Transformation of time,

9. Autotelic experience.

Responses were measured on a 5-point Likert scale ranging from strongly disagree (1) to strongly agree (5). Examples of the FSS include "I felt in total control of what I was doing" and "I really enjoyed the experience." Responses were summed for scores within each subscale, and then all subscales were summed for a total FSS score. Subscale scores ranged from 4 to 20 and total FSS scores ranged from 36 to 180 , with higher scores representing a higher experience of flow within both the subscales and the total flow state scores (Jackson, Eklund, \& Martin 2009). The inter-item reliability of the total FSS scale was strong $(\alpha=0.83)$, as well as each of the nine subscales $(\alpha>0.80)$. Furthermore, the construct validity of the FSS has been shown to be strong, ranging from 0.177 to 0.724 (median $r=0.50$; Cosma 1999). Permission to use the FSS was granted by Mind Garden, Inc. (Jackson et al. 2009). 


\section{Data Analysis}

Multiple linear regression was used to examine the association between the type of athlete and experience of flow. Both unadjusted and adjusted models were computed. For each model, both gender (i.e., male or female) and the year in school (i.e., freshman, sophomore, junior, senior) were controlled to further show the significance of the sport being played in the flow state. Additionally, differences between the sport setting and its effect on flow were examined within each subscale using multiple linear regression. Within the FSS, the nine dimensions (subscales) of flow were also analyzed for differences between individual and team athletes. For all statistical analyses, the level of significance was $p<0.05$. The researchers used the statistical program R (version 3.1.1) for all statistical analyses.

\section{Results}

\section{Total Flow State Scores}

Table 2 shows the models for the relationship between mean flow scores and type of sport (i.e., individual vs. team). Individual sports had a significantly lower mean flow score compared to team sports $(\beta$ $=-1.66, p=0.004$, model 1).

Table 2.

Association between type of sport on dimensions of flow

\begin{tabular}{|c|c|c|c|}
\hline Flow Model & $\beta$ & $p$-value & $\mathrm{R}^{2}$ \\
\hline \multicolumn{4}{|l|}{ Model 1: Total Flow } \\
\hline Individual Sport & -1.66 & 0.04 & 0.03 \\
\hline Team Sport & Reference & & \\
\hline \multicolumn{4}{|c|}{ Model 2: Challenge-Skill Balance } \\
\hline Individual Sport & -0.15 & 0.09 & 0.01 \\
\hline Team Sport & Reference & & \\
\hline \multicolumn{4}{|c|}{ Model 3: Merging of Action and Awareness } \\
\hline Individual Sport & -0.38 & 0.01 & 0.09 \\
\hline Team Sport & Reference & & \\
\hline \multicolumn{4}{|l|}{ Model 4: Clear Goals ${ }^{\dagger}$} \\
\hline Individual Sport & -0.05 & 0.33 & 0.01 \\
\hline Team Sport & Reference & & \\
\hline \multicolumn{4}{|c|}{ Model 5: Unambiguous Feedback } \\
\hline Individual Sport & -0.24 & 0.08 & 0.02 \\
\hline Team Sport & Reference & & \\
\hline \multicolumn{4}{|c|}{ Model 6: Concentration on Task at Hand } \\
\hline Individual Sport & -0.33 & 0.01 & 0.04 \\
\hline Team Sport & Reference & & \\
\hline \multicolumn{4}{|c|}{ Model 7: Sense of Control ${ }^{*}$} \\
\hline Individual Sport & -0.28 & 0.05 & 0.06 \\
\hline Team Sport & Reference & & \\
\hline \multicolumn{4}{|c|}{ Model 8: Loss of Self-Consciousness* } \\
\hline Individual Sport & 0.13 & 0.42 & 0.03 \\
\hline Team Sport & Reference & & \\
\hline \multicolumn{4}{|c|}{ Model 9: Transformation of Time } \\
\hline Individual Sport & -0.18 & 0.27 & 0.04 \\
\hline Team Sport & Reference & & \\
\hline \multicolumn{4}{|l|}{ Model 10: Autotelic } \\
\hline Individual Sport & -0.15 & 0.11 & 0.01 \\
\hline Team Sport & Reference & & \\
\hline
\end{tabular}


Note: Bold indicates statistically significant $(p<0.05)$

${ }^{\dagger}$ Controlled for gender (male, female)

*Controlled for race (African American, Caucasian, Other)

'Controlled for year in school (freshman, sophomore, junior, senior)

Source: Own study.

\section{Dimensions of Flow}

The type of sport was a significant predictor of three of the nine dimensions of flow $(p<0.05)$, with an additional two dimensions approaching significance $(p<0.09)$ (see Table 2$)$. In the model, individual sports had a significantly lower score in each dimension, except for the Loss of Self-Consciousness dimension. The largest difference between team and individual sports was the Action-Awareness Merging dimension $(\beta=-$ $0.38, p=0.01$, model 3), explaining approximately $9 \%$ of the variance. The Challenge-Skill Balance $(\beta=-$ $0.15, p=0.09$, model 2) and Unambiguous Feedback $(\beta=-0.24, p=0.08$, model 5) dimensions both approached significance with a higher team athlete score. The smallest difference between team and individual sports was Concentration on Task at Hand $(\beta=-0.33, p=0.01$, model 6$)$, explaining approximately $4 \%$ of the total variance. The Sense of Control dimension was also significantly higher in the team athletes $(\beta=-0.28, p$ $=0.05$, model 7 ), explaining approximately $6 \%$ of the variance.

\section{Discussion}

The purpose of this study was to examine if there was a difference in the experience of flow within athletes participating in individual sports and those participating in team sports by using the FSS (Jackson \& Marsh 1996). From the data, there was evidence suggesting that team athletes and individual athletes experience total flow differently. The results also showed significant differences among three of the nine dimensions, with an additional two approaching significance, as team athletes reported experiencing flow at a higher level than individual athletes. The results indicated that flow was experienced by team athletes significantly more than individual athletes. The regression analysis also showed that the specific sport explained a substantial amount of the variance in total flow (approximately 4\%) and within several dimensions (up to 9\%). This adds meaning to the results of individual sport athletes and their experience of flow as compared to team sport athletes. This result differs from Russell (2001), who found no significant differences and stated that flow was experienced similarly across all sport settings. In addition, experiencing flow has traditionally only been associated with individual sports (Bakker et al. 2011). Due to the dearth of current research, future studies should continue to explore this trend across different populations and sports.

The results also found significance among three of the nine flow dimensions (and two others approaching significance), specifically the dimensions that team athletes are theorized to experience more than individual athletes (i.e., action-awareness merging, concentration on the task at hand, sense of control, challenge skills balance, and unambiguous feedback). There are some probable reasons for why these dimensions were experienced more often in the team sport setting than the individual sport setting. For the dimensions of challenge-skills balance and unambiguous feedback, the athletic challenge may be more recognizable in a team sport since intra-squad competition is more readily available to team sport athletes than it is to individual sport athletes. In addition, the athletes may have reported their experience as "more enjoyable" due to the increased social interactions on a team. However, it was surprising that the team athletes experienced a greater sense of control and concentration. It was expected that individual athletes would score higher in these dimensions because they would have greater autonomy and fewer distractions than team athletes. Additional research is needed to explore the different dimensions among different sport settings.

It is common for athletes to not experience all the dimensions of flow. Similar to the results of the present study, Jackson (1996) found that $80 \%$ of elite athletes (team and individual athletes) reported experiencing the dimensions of action-awareness merging, concentration on the task at hand, and sense of control more so than the other six subscales. Jackson (1996) hypothesized that these three dimensions may be more central to elite athletes in flow. The current study's results support this conclusion with college athletes. Additionally, Jackson (1996) also found that two dimensions, transformation of time and loss of self-consciousness, were not universally endorsed. The current study concurs with those findings because there were no statistically 
significant differences between team athletes or individual athletes across those two dimensions. Finally, the dimensions of challenge-skill balance and clear goals were reported just over a third of the time by Jackson (1996). However, the current study showed a higher level of the challenge-skill balance dimension within team athletes, and while not significant among this sample at $p<0.05$, our model suggests an approach to significance at $p=0.09$. Jackson's study (1996) involved elite athletes, whereas this study examined collegiate athletes. It is possible that the college athletes in the current study were not at the skill level of the elite athletes studied by Jackson, thus causing the challenge-skill balance to be more likely in this study. Thus, the skill level of the athletes should be explored further within future flow research.

Similarly, Cosma (1999) found four of the nine flow dimensions to be more significant (i.e., concentration, autotelic experience, clear goals, and unambiguous feedback) among a combined athlete pool consisting of both team and individual sports. In the current study, concentration was significant and unambiguous feedback approached significance, while clear goals and autotelic experience did not. This result is unusual because within a team setting it would be expected that having clear goals would facilitate flow. However, our results suggest that this does not differ between team and individual athletes. Additional research is needed to investigate the role of goal setting and communication among team athletes in different sport settings.

\section{Practical Application}

The application of these findings for both coaches and athletes is significant. Specifically, within the team sport setting, these findings suggest that the flow state can and does exist. Facilitating flow among the athletes on a team can elicit the idea of "team flow," where certain players can get into flow and then act as a catalyst for the entire team to experience flow (Swann et al. 2012). However, the idea of team flow warrants future research because it could help explain exceptional team performances and would contribute to a better understanding of flow. In addition, future research can explore how flow can then affect the outcome of a game, match, or event.

To help elicit the flow state, coaches of all sports should focus more on psychological training, as this type of training has been shown to facilitate the flow state (Kaufman, Glass, \& Arnkoff 2009; Koehn, Morris, $\&$ Watt 2014). For example, imagery intervention has shown to increase flow experiences (Koehn et al. 2014), as has an evaluation of mindful sport performance enhancement (Kaufman et al. 2009). Coaches can also nurture flow state during practice, which could also increase the likelihood of flow occurring in a game or competition. This can be done by creating practice situations that mimic game demands, which can foster opportunities for athletes to manipulate their game-like experience, potentially encouraging flow states. Team athletes would have more opportunities to achieve a "rhythm" or "confidence" in their performance through game-like exposure in practice, thus leading to a greater possibly of experiencing flow. Teaching psychological skills and fostering situations to encourage flow are important because there is evidence that athletes believe the flow state is controllable (Chavez 2008; Russell 2001). However, to date, studies about learning to control flow are rare. Further research should investigate the controllability of flow.

Although the study strived to include a diverse sample of sports and athletes, it is not without limitations. First, this study included a self-report of a previous experience of the flow state based on memory. The illusiveness and unpredictability of the flow state make it nearly impossible to measure during the moment, so current research must rely on memory recall. Future research should explore more objective ways to measure flow. Similarly, it was unknown how long ago the flow state occurred for the athlete and how accurate the memory of the experience was at the time of the report. Future research should also take into consideration the length of time an athlete has participated in the sport because athletes can experience some sports at a younger age than others, likely increasing the chance of experiencing flow.

Strengths of this study include the sample size $(\mathrm{N}=104)$ and the diversity of the population. The sample size shows that the study was adequately powered and is more generalizable than results from a smaller sample. Additionally, this exploratory study compared the experiences of flow between team and individual athletes and is one of the only studies to date to answer the repeated call in the literature, adding to the nascent body of 
research on team athletes and the flow experience. In summary, the results showed that athletes from team sports experienced total flow at a higher overall rate than individual sport athletes and scored significantly higher within three of the nine subscales of flow. This study also suggested that flow is experienced across different sport settings. From the results, it is clear that team sport athletes can experience flow at the same level as individual sport athletes. Coaches and athletes should consider these results and implications as they relate to practice and preparation for competitions.

\section{REFERENCES}

Bakker, A.B., Oerlemans, W., Demerouti, E., Slot, B., \& Ali, D. (2011). Flow and performance: A study among talented Dutch soccer players. Psychology of Sport \& Exercise, 12(4), 442-450. DOI: 10.1016/j.psychsport.2011.02.003

Chavez, E.J. (2008). Flow in sport: A study of college athletes. Imagination, Cognition, and Personality, 28(1), 69-91. DOI: $10.2190 /$ IC.28.1.f

Cohen, J. (1988). Statistical Power Analysis for the Behavioral Sciences (2 ${ }^{\text {nd }}$ ed.). Hillsdale, NJ: Erlbaum.

Cosma, J.B. (1999). Flow in teams. Doctoral dissertation, The Chicago School of Professional Psychology, Chicago, IL. Retrieved from Springfield College through Interlibrary loan.

Csikszentmihalyi, M. (1990). Flow: The Psychology of Optimal Experience. New York, NY: Harper \& Row.

Faul, F., Erdfelder, E., Buchner, A., \& Lang, A.-G. (2013). G*Power Version 3.1.7 [computer software]. Kiel, Germany: Uiversität Kiel. Retrieved from http://www.psycho.uni-duesseldorf.de/abteilungen/aap/gpower3/download-and-register

Jackson, S.A. (1996). Toward a conceptual understanding of the flow experience in elite athletes. Research Quarterly for Exercise and Sport, 67(1), 76-89. DOI: 10.1080/02701367.1996.10608859

Jackson, S.A., Eklund, R.C., \& Martin A.J. (2009). The Flow Scales Instrument and Scoring Guide. Menlo Park, CA: Mind Garden, Inc. Permission granted for use on May 9, 2016.

Jackson, S.A. \& Marsh, H.W. (1996). Development and validation of a scale to measure optimal experience: The Flow State Scale. Journal of Sport \& Exercise Psychology, 18(1), 17-35. DOI: 10.1123/jsep.18.1.17

Jackson, S. \& Roberts, G.C. (1992). Positive performance states of athletes: Toward a conceptual understanding of peak performance. The Sport Psychologist, 6, 156-171. DOI: 10.1123/tsp.6.2.156

Kaufman, K.A., Glass, C.R., \& Arnkoff, D.B. (2009). Evaluation of mindful sport performance enhancement (MSPE): A new approach to promote flow in athletes. Journal of Clinical Sport Psychology, 3(4), 334-356. DOI: 10.1123/jesp.3.4.334

Koehn, S., Morris, T., \& Watt, A.P. (2014). Imagery intervention to increase flow state and performance in competition. Sport Psychologist, 28(1), 48-59. DOI: 10.1080/1612197X.2016.1187653

Qualtrics. (2015). Provo, UT. http://www.qualtrics.com.

Privette, G. (1983). Peak experience, peak performance, and flow: A comparative analysis of positive human experiences. Journal of Personality and Social Psychology, 45, 1361-1368. DOI: 10.1037/0022-3514.45.6.1361

Privette, G. \& Bundrick, C.M. (1991). Peak performance, peak experience and flow: Personal descriptions and theoretical constructs. Journal of Social Behaviour and Personality, 6, 169-188.

Russell, W.D. (2001). An examination of flow state occurrence in college athletes. Journal of Sport Behavior, 24(1), 83105 .

Stavrou, N.A., Jackson, S.A., Zervas, Y., \& Karteroliotis, K. (2007). Flow experience and athletes' performance with reference to the Orthogonal Model of Flow. Sport Psychologist, 21(4), 438-457. DOI: 10.1123/tsp.21.4.438

Swann, C., Keegan, R.J., Piggott, D., \& Crust, L. (2012). A systematic review of the experience, occurrence, and controllability of flow states in elite sport. Psychology of Sport \& Exercise, 13(6), 807-819. DOI: 10.1016/j.psychsport.2012.05.006

Swann, C. (2016). Flow in sport. In L. Harmat, F. Ullen, G. Sadlo, F.O. Andersen, \& J. Wright (Eds.), Flow Experience: Empirical Research and Applications (pp. 51-64). Switzerland: Springer International Publishing. 
AUTHOR'S ADDRESS: Joni M. Boyd

PESH Department

Winthrop University

216 L West Center

Rock Hill, SC 29732

E-mail:boydj@winthrop.edu

Received: 13 March 2018; Accepted: 30 April 2018 